\title{
MÉTODOS DE BAIXO CUSTO PARA PURIFICAÇÃo DE REAGENTES E CONTROLE DA CONTAMINAÇÃO PARA A DETERMINAÇÃO DE METAIS TRAÇOS EM ÁGUAS NATURAIS
}

\author{
Maria Lúcia A. M. Campos*\#, Anderson Bendo e Fabíola C. Viel \\ Departamento de Química, Universidade Federal de Santa Catarina, CP 476, 88040-900 Florianópolis - SC
}

Recebido em 11/7/01; aceito em 7/1/02

\begin{abstract}
LOW COST METHODS TO PURIFY LOW GRADE REAGENTS AND TO CONTROL CONTAMINATION FOR TRACE METAL DETERMINATION IN NATURAL WATERS. Probably one of the most difficult and challenging aspects of measuring trace metals in natural waters is to avoid contamination during sampling, manipulation and analysis. This work discusses how to avoid contamination using simple procedures, and considers alternative methods to purify deionised water and low grade reagents to enable accurate determination of trace metals in natural waters in a common laboratory. Measurements were performed by cathodic stripping voltammetry and copper was used as a model metal to test the procedures. It was possible to evaluate copper speciation in natural waters even when total dissolved copper concentration was as low as $1.5 \mathrm{nmol} \mathrm{L}^{-1}$. The methods' accuracy was confirmed by analysis of certified seawater.
\end{abstract}

Keywords: copper; speciation; cathodic stripping voltammetry.

\section{INTRODUÇÃO}

Apesar da preocupação com o meio ambiente ter surgido de modo significativo há apenas poucas décadas, dia após dia, esta preocupação vem tomando novas dimensões dentro dos mais diversos setores da sociedade mundial. A importância da preservação dos recursos hídricos tem levado à necessidade de monitorar e controlar a contaminação destes ambientes, e os metais pesados estão entre os contaminantes mais tóxicos e persistentes do ambiente aquático. Portanto, suas fontes, transporte e destino precisam ser avaliados.

Visto que os organismos aquáticos tendem a acumular metais pesados e contaminantes orgânicos em seus tecidos, mesmo quando a água possui níveis desses compostos abaixo da concentração máxima tolerada pela legislação, há grandes riscos de contaminação dentro da cadeia trófica. No caso de peixes, a ingestão de alimentos e água é a rota principal de entrada de contaminantes nesses organismos. Animais filtradores como os mexilhões, filtram vários litros de água por hora, e consequentemente podem concentrar de 10 a $10^{5}$ vezes vários contaminantes em seus tecidos, com relação à água do mar'. Se a fração tóxica de um metal encontrada num corpo d'água for alta o suficiente para inibir o crescimento de apenas uma parte da comunidade planctônica, isto pode acarretar no comprometimento de toda a cadeia trófica, levando-se em conta a bioacumulação e os efeitos crônicos que o metal pode causar.

Uma das menores concentrações de metais pesados na natureza é encontrada nas águas oceânicas ${ }^{2}$. Mesmo após o desenvolvimento de instrumentos, métodos analíticos suficientemente sensíveis e reagentes ultra purificados para a análise de metais traços $\left(<50 \mathrm{nmol} \mathrm{kg}^{-1}\right)^{3}$ nessas águas, a contaminação das amostras impedia a obtenção de dados coerentes sob o ponto de vista biogeoquímico. Somente após a conscientização de que a amostragem, preservação

\footnotetext{
* e-mail: lcampos@ffclrp.usp.br

\# endereço atual: Departamento de Química, Faculdade de Filosofia, Ciências e Letras de Ribeirão Preto, Universidade de São Paulo, Av. Bandeirantes, 3900, 14040-901 Ribeirão Preto- SP
}

e manuseio das amostras antes da análise podiam representar maiores fontes de erro que a própria análise química, é que foram publicados, em 1977 e em 1981, os primeiros dados confiáveis da distribuição de cobre e chumbo, respectivamente, em águas de oceano aberto $^{4,5}$. Tendo em mãos métodos extremamente sensíveis e mantendo o controle da contaminação, foi então possível dar início aos estudos de especiação química de metais, e assim, verdadeiramente avaliar a toxicidade de distintas espécies químicas para os organismos aquáticos ${ }^{6}$. A necessidade de utilizar procedimentos limpos na determinação de metais traços não se restringe somente ao ambiente marinho, mas também são imprescindíveis nos estudos envolvendo águas de rios, lagos e estuários, onde pode haver concentrações mais elevadas de metais ${ }^{7,8}$.

$\mathrm{O}$ estudo da biogeoquímica de metais traços e sua especiação química em ambientes aquáticos naturais no Brasil é bastante recente, tendo em vista a sofisticação de algumas técnicas e o alto custo da instrumentação e dos reagentes ultra-puros. A literatura que aborda a prevenção da contaminação e análise de metais traços, na sua grande maioria se refere ao uso de laboratórios limpos classe 100 que, portanto, possuem sistemas de filtração de ar, bancadas de fluxo laminar, superfícies especiais, além de contar com a disponibilidade de reagentes ultra-puros ${ }^{7-10}$; enfim, condições que não são facilmente encontradas em laboratórios de pesquisa no Brasil. Portanto, este trabalho tem como objetivos discutir métodos simples para minimizar a contaminação por metais em laboratório comum; demonstrar a necessidade de observar alguns cuidados de manuseio, que são indispensáveis para análise de metais no nível de traço, e divulgar alguns métodos de baixo custo para purificação de reagentes, que venham ser compatíveis com a realidade de muitas instituições de pesquisa no Brasil. O metal cobre foi escolhido como modelo para testar os métodos de controle da contaminação e purificação de reagentes por ser um metal abundante no ambiente e, portanto, o controle da contaminação durante os procedimentos de manuseio e análise das amostras requer grande rigor. Para a avaliação do cobre foi utilizada a voltametria de redissolução catódica que é uma técnica de grande sensibilidade ${ }^{11}$ e de custo relativamente baixo, tanto para compra do equipamento como para sua manutenção e uso. 


\section{PARTE EXPERIMENTAL}

\section{Reagentes}

As soluções de cobre utilizadas foram obtidas após diluições sucessivas do padrão de 1000 ppm SpectrosoL, Merck e preservadas em pH 2 (HCl-purificado) em geladeira. A solução estoque de 0,05 mol L ${ }^{-1}$ salicilaldoxime (SA; Sigma) foi preparada em $0,6 \mathrm{~mol} \mathrm{~L}^{-1}$ $\mathrm{HCl}$ (Quimex) purificado, e a solução estoque de oxina $0,2 \mathrm{~mol} \mathrm{~L}^{-1}$ (AnalaR, BDH) em 0,2 mol L-1 $\mathrm{HCl}$ (Quimex) purificado. A solução tampão consistiu de $1 \mathrm{~mol} \mathrm{~L}^{-1} \mathrm{H}_{3} \mathrm{BO}_{3}$ (AristaR, BDH) e $0,4 \mathrm{~mol} \mathrm{~L}-1$ $\mathrm{NH}_{3}$ (Quimex) purificada. Os reagentes $\mathrm{MnCl}_{2}, \mathrm{KMnO} 4$ e $\mathrm{NaOH}$ foram de grau analítico.

A suspensão de $\mathrm{MnO}_{2}$ foi preparada misturando $100 \mathrm{~mL}$ de $\mathrm{KMnO}_{4} 10^{-2} \mathrm{~mol} \mathrm{~L}^{-1}$ e $100 \mathrm{~mL}$ de $\mathrm{NaOH} 2 \times 10^{-2} \mathrm{~mol} \mathrm{~L}^{-1}$ em erlenmeyer, com a adição lenta de $100 \mathrm{~mL}$ de $\mathrm{MnCl}_{2} 1,5 \times 10^{-2} \mathrm{~mol} \mathrm{~L}^{-1}$ sob agitação, para evitar a formação de flocos muito grandes de $\mathrm{MnO}_{2}$. A suspensão de $\mathrm{MnO}_{2}$ foi centrifugada durante 30 min usando $4000 \mathrm{rpm}$, e o sobrenadante desprezado. O precipitado foi lavado com água desionizada, centrifugado mais 2 vezes, e finalmente ressuspenso em $250 \mathrm{~mL}$ de água desionizada para atingir a concentração de $10^{-2} \mathrm{~mol} \mathrm{~L}^{-1}$.

Neste trabalho, o termo "água desionizada" é utilizado de forma simplificada para se referir à água destilada em sistema de quartzo e posteriormente desionizada por sistema de troca iônica (marca Permution), e "água ultra-pura" é aquela que foi primeiramente destilada em sistema de quartzo, e posteriormente desionizada em sistema Milli- $\mathrm{Q}^{\mathrm{TM}}$ (Millipore >18 M $\Omega$ ).

\section{Determinação de cobre e equipamentos}

As análises de cobre foram realizadas por voltametria de redissolução catódica usando alíquotas de $10 \mathrm{~mL}$ de amostra, contendo $0,01 \mathrm{~mol} \mathrm{~L}^{-1}$ de tampão borato (pH 8,3) e $25 \mu \mathrm{mol} \mathrm{L} \mathrm{L}^{-1} \mathrm{SA}$, de acordo com o método descrito por Campos e van den Berg ${ }^{12}$. As amostras que foram analisadas utilizando o ligante oxina $\left(20 \mu \mathrm{mol} \mathrm{L} \mathrm{L}^{-1}\right)^{13}$ são identificadas no corpo do trabalho. As amostras acidificadas com $\mathrm{HCl}$ ( $\mathrm{pH}$ 2) tiveram seu $\mathrm{pH}$ neutralizado com uma solução de $\mathrm{NH}_{3}$ antes da análise. O tempo de pré-concentração do metal foi de 1 ou $2 \mathrm{~min}$, dependendo da concentração do metal na amostra. O potenciostato utilizado foi um modelo PAR 263A e o sistema de eletrodos modelo PAR 303A com eletrodo de $\mathrm{Hg}$ de gota pendente, eletrodo de $\mathrm{Ag} /$ $\mathrm{AgCl}(\mathrm{KCl} 3 \mathrm{M})$ e eletrodo de platina. A destruição da matéria orgânica para avaliação da concentração de cobre dissolvido total em amostras naturais foi realizada em reator de UV durante $4 \mathrm{~h}$ de acordo com Campos e colaboradores ${ }^{14}$. O potenciômetro utilizado foi da marca Orion, modelo 720A, com eletrodo de vidro combinado Orion.

\section{Cuidados iniciais}

Todas as bancadas do laboratório foram revestidas com plástico e os materiais utilizados na análise de metais foram lavados e armazenados em 2 sacos plásticos (um interno limpo, e outro externo mais vulnerável à contaminação). As células voltamétricas e tubos de quartzo foram mantidos em banhos de $\mathrm{HCl} 1 \mathrm{~mol} \mathrm{~L}^{-1}$ tampados, sendo bem enxaguados com água desionizada antes de utilizados. Todas as ponteiras de pipeta foram lavadas com $\mathrm{HCl} 1 \mathrm{~mol} \mathrm{~L}^{-1}$, sendo que depois de enxaguadas com água desionizada foram armazenadas em tubos de poliestireno etiquetados e com tampa, para que as ponteiras somente fossem reutilizadas para a mesma solução.

As soluções utilizadas na análise de metais foram preparadas em balança analítica diretamente nos frascos de polietileno onde foram armazenadas. $\mathrm{O}$ procedimento consistiu em pesar aproximadamente a massa desejada e depois pesar exatamente a água necessária usando uma pisseta. $\mathrm{O}$ fato de serem utilizados microlitros de reagentes em cada análise, evita a preparação de grandes quantidades destes. As diluições dos reagentes também foram realizadas em balança analítica usando micropipeta e pisseta para ajustar o volume final da solução (por peso). O volume da amostra filtrada também foi calculado por diferença de peso e assim foi possível calcular o volume de ácido a ser adicionado para atingir pH 2. Balão volumétrico, ou qualquer outro tipo de vidraria, não foi utilizado para minimizar o risco de contaminação dos reagentes, e somente foram utilizadas espátulas e pinças de plástico pré-lavadas.

No momento da preparação da alíquota para análise, a célula voltamétrica enxaguada (com auxílio de uma pinça) foi colocada para escorrer em lenço de papel limpo e em seguida foi mantida tampada com placa de Petri de poliestireno. A adição da amostra e dos reagentes foram realizadas rapidamente, permitindo o mínimo de tempo possível de contato com o ar. Após a análise de uma alíquota, o conjunto de eletrodos foi lavado com água desionizada, $\mathrm{HCl} 1 \mathrm{~mol} \mathrm{~L}^{-1} \mathrm{e}$ novamente com água desionizada antes de introduzir rapidamente a próxima alíquota. A célula voltamétrica usada foi lavada da mesma forma que os eletrodos e retornada ao banho ácido.

Para qualquer manuseio de reagentes ou material utilizados na análise de metais traços, foram utilizadas luvas descartáveis de polietileno limpas (encontradas no comércio local). As luvas cirúrgicas (nunca usar com talco) têm as desvantagens de serem muito mais caras e difíceis de por e tirar, sendo que as largas luvas de polietileno, uma vez usadas, podem ser armazenas em saco plástico limpo e reutilizadas algumas vezes mais, desde que seja para o mesmo procedimento. O processo de fabricação de luvas de borracha (como por exemplo, aquelas para uso doméstico) utiliza grandes quantidades de metais e, portanto, seu uso deve ser evitado ${ }^{15}$. Vale a pena lembrar que as luvas estão tão limpas quanto o último objeto tocado, e ao vesti-las, as mãos nuas não podem tocar no exterior das mesmas.

\section{Lavagem do material}

Com o objetivo de remover resíduos orgânicos, os frascos de polietileno de alta densidade foram primeiramente lavados com água corrente, detergente comercial diluído e escova. Posteriormente, os frascos foram enxaguados, preenchidos completamente com detergente $\sim 5 \%(\mathrm{v} / \mathrm{v})$, e deixados em repouso por uma semana em bandeja plástica limpa envolvida com saco plástico. Preferencialmente devese deixar os frascos imersos em banhos preparados em recipientes plásticos com tampa, tanto de detergente quanto de ácido, pois a limpeza externa do material é tão importante quanto a interna ${ }^{15}$.

Após o período em detergente, os frascos foram enxaguados exaustivamente com água corrente (pelo menos 5 vezes, e com muita água), 3 vezes com água desionizada, preenchidos completamente com $\mathrm{HNO}_{3} 5 \mathrm{~mol} \mathrm{~L}^{-1}$ e deixados em bandeja envolvida com plástico por uma semana. Posteriormente, os frascos foram enxaguados 5 vezes com água desionizada e preenchidos completamente com esta mesma água (havendo disponibilidade, os frascos devem ser preenchidos com água ultra-pura). Cada frasco foi ensacado individualmente e, posteriormente, ensacados novamente em grupos de 3 ou 4. Durante todo o processo de limpeza, tomou-se o cuidado de nunca colocar os frascos e tampas diretamente sobre a bancada, mas sempre dentro da bandeja apropriada.

Statham ${ }^{15}$ sugere o uso adicional de um banho em $\mathrm{HCl}$ 1:1 (v/v) por uma semana antes do banho em $\mathrm{HNO}_{3}$ também 1:1 (v/v). Cam$\operatorname{pos}^{16}$ e Achterberg ${ }^{17}$ utilizaram banhos de $\mathrm{HCl} 6 \mathrm{~mol} \mathrm{~L}^{-1}$, e depois $\mathrm{HNO}_{3} 2 \mathrm{~mol} \mathrm{~L}^{-1}$, com posterior adição de $\mathrm{HCl}$ purificado para atingir pH 2 na água ultra-pura estocada nos frascos. Há uma gama de vari- 
ações propostas quanto aos procedimentos de limpeza, porém, a eficiência do processo de limpeza simplificado descrito neste trabalho foi confirmada pela realização de testes de brancos nas águas armazenadas nesses frascos (ver seção "Branco de reagentes e limite de detecção").

Os frascos não precisam ser secados, mesmo que isto seja realizado em bancada de fluxo laminar, pois no momento da coleta da amostra, cada frasco é enxaguado pelo menos duas vezes com a própria água a ser coletada, e qualquer resíduo, se ainda presente, deve ser eliminado nesse processo ${ }^{16,17}$. No caso da coleta de água de chuva, os frascos podem ser simplesmente bem esvaziados antes de colocados no coletor, visto que tanto a possibilidade de contaminação com os resíduos, como a possibilidade de diluição da amostra são muito pouco prováveis.

\section{Amostragem}

Ambientes com águas calmas freqüentemente permitem o uso de embarcações pequenas e portanto, a amostragem de águas superficiais pode ser feita manualmente submergindo os frascos. É interessante amostrar alguns centímetros abaixo da superfície para evitar a coleta seletiva da micro-camada rica em matéria orgânica e metais pesados, que portanto possui características distintas do restante da camada de superfície do ambiente aquático em estudo ${ }^{18}$. Como as luvas são geralmente curtas, deve-se tomar o cuidado de não deixar entrar água nas mãos, tornando-as uma fonte de contaminação. Portanto, pode ser preferível usar um saco plástico grande que cubra desde a mão até o antebraço, prendendo o mesmo com um elástico. É recomendável que a amostragem seja feita por duas pessoas, uma que manuseia o saco externo dos frascos e outros materiais 'sujos', enquanto a outra pessoa veste as luvas no momento da coleta e manuseia somente os materiais 'limpos'. As amostras devem ser preservadas resfriadas durante o trabalho de campo para evitar a dessorção de metais do material particulado para a solução.

As amostras devem ser coletadas contra a corrente, isto é, antes que a água a ser coletada atinja o barco e seja contaminada pelo mesmo. Amostras de águas profundas podem ser coletadas utilizando garrafas do tipo Niskin pré-lavadas com soluções ácidas, porém um método mais simples e menos suscetível à contaminação é utilizar uma bomba peristáltica à bateria, acoplada a tubos de polietileno também pré-lavados ${ }^{19}$. A água, de uma profundidade desejada, é bombeada para dentro do barco, podendo ser filtrada por um sistema de filtração adaptado na ponta do tubo que está emersa, proporcionando assim o armazenamento da amostra diretamente no frasco onde será preservada. Qualquer tipo de lastro utilizado deve ser pré-lavado e livre de contaminação metálica.

\section{RESULTADOS E DISCUSSÃO}

\section{Purificação da água}

A água para a preparação dos reagentes deve ser ultra-pura, mas nem sempre é possível ter disponível um sistema de desionização do tipo Milli-Q ou similar. Portanto, um método muito simples e barato para purificar a água destilada e/ou desionizada é utilizar uma suspensão de $\mathrm{MnO}_{2}$, que tem a propriedade de adsorver de forma muito eficiente os íons metálicos.

Para testar a eficiência do método, foram preparadas diversas soluções de cobre em água ultra-pura (Tabela 1). Para um volume de $200 \mathrm{~mL}$ de cada solução de cobre, foram adicionados $2,0 \mathrm{~mL}$ da suspensão de $\mathrm{MnO}_{2} 10^{-2} \mathrm{~mol} \mathrm{~L}^{-1}$. As soluções foram então agitadas durante $10 \mathrm{~h}$ em mesa agitadora e posteriormente deixadas em repouso por $2 \mathrm{~h}$. A filtração foi realizada em sistema de polipropileno
Tabela 1. Concentração de cobre em água ultra-pura contaminada com cobre, antes e após o tratamento com a suspensão de $\mathrm{MnO}_{2}$ $10^{-4} \mathrm{~mol} \mathrm{~L}^{-1}$

\begin{tabular}{ccc}
\hline \multicolumn{2}{c}{$[\mathrm{Cu}]\left(\mathrm{nmol} \mathrm{L}^{-1}\right)$} & \% Remoção \\
\cline { 1 - 2 } antes tratamento & após tratamento & \\
\hline 1000 & 30,0 & 97 \\
500 & 10,0 & 98 \\
2,5 & 0,33 & 87 \\
\hline
\end{tabular}

de $25 \mathrm{~mm}$ de diâmetro, de acordo com o procedimento descrito abaixo, e a concentração de cobre no filtrado foi avaliada. Com apenas um tratamento foi possível remover 97 e $98 \%$ do cobre presente nas soluções de 1000 e $500 \mathrm{nmol} \mathrm{L}^{-1} \mathrm{Cu}$, respectivamente, porém, levando a concentrações de cobre ainda elevadas. A eficiente purificação de uma solução contendo $2,5 \mathrm{nmol} \mathrm{L}{ }^{-1} \mathrm{Cu}$ demonstra que se for necessário, é possível chegar em concentrações satisfatórias de metal com tratamentos sucessivos.

\section{Purificação do $\mathrm{HCl}, \mathrm{NH}_{3}$ e tampão}

O alto custo dos reagentes ultra-puros, e dos destiladores de quartzo utilizados na purificação de ácidos concentrados pode ser grande obstáculo para aqueles que desejam analisar metais no nível de traços. Porém, a alta pressão de vapor do ácido clorídrico e da amônia permitem a purificação destes reagentes por destilação isotérmica, como ilustra a Figura 1a. Neste trabalho, o "destilador" consistiu de um recipiente plástico do tipo "tupperware" transparente e com tampa de cor branca. O uso de plástico transparente é sempre preferível, visto que os pigmentos coloridos são ricos em metais ${ }^{15}$. Após a lavagem do recipiente como descrito anteriormente, este foi colocado dentro de um grande saco plástico limpo, e durante todo o manuseio foram utilizadas luvas descartáveis limpas. Dentro do "destilador", devidamente ensacado, foram introduzidos um béquer pré-lavado com cerca de $30 \mathrm{~mL}$ da solução concentrada de $\mathrm{NH}_{3}($ ou $\mathrm{HCl})$ da marca Quimex, e um frasco de polietileno de alta densidade de boca larga de $60 \mathrm{~mL}$ (também pré-lavado) contendo cerca de $50 \mathrm{~mL}$ de água previamente purificada. $\mathrm{O}$ "destilador" foi bem fechado com tampa, permitindo que a base volatilizada (ou ácido) saturasse a atmosfera interna do recipiente e por difusão, dissolvesse na água purificada. A solução concentrada foi trocada 3 vezes por uma nova alíquota a cada 2 dias, para acelerar o processo de destilação. Após 8 dias, o frasco contendo a solução purificada foi bem fechado, retirado do sistema, e ensacado 2 vezes com sacos plásticos limpos.

A Figura 1b ilustra a titulação potenciométrica de uma solução de $\mathrm{NH}_{3}$ destilada utilizando uma alíquota de $20 \mu \mathrm{L}$ desta solução, diluída em $25 \mathrm{~mL}$ de água ultra-pura, e titulada com uma solução padronizada de $\mathrm{HCl} 9,9910^{-2} \mathrm{~mol} \mathrm{~L}^{-1}$. A concentração da solução purificada de $\mathrm{NH}_{3}$ foi de $8,8 \mathrm{~mol} \mathrm{~L}^{-1}$, e de $\mathrm{HCl}$ de $7,7 \mathrm{~mol} \mathrm{~L}^{-1}$, o que demonstra a boa eficiência do processo de destilação isotérmica. A pureza dos reagentes foi verificada por meio da análise de brancos apresentada na seção "Branco de reagentes e limite de detecção".

A solução de tampão borato utilizada neste trabalho foi preparada com $\mathrm{H}_{3} \mathrm{BO}_{3}$ de alta pureza (AristaR, BDH) e somente a purificação da $\mathrm{NH}_{3}$ foi necessária. Caso seja necessário purificar a solução de tampão, Campos ${ }^{16}$ mostrou que o uso da resina Chelex-100 é bastante eficiente, atingindo concentrações de cobre abaixo do limite de detecção do método $\left(0,1 \mathrm{nmol} \mathrm{L^{-1 }}\right.$ para 1 min de pré-concentração usando SA). A resina foi inicialmente convertida para a forma amoniacal submergindo a mesma em acetato de amônio $0,1 \mathrm{~mol} \mathrm{~L}^{-1}$ ( $24 \mathrm{~h})$ e posteriormente lavando-a com água ultra-pura para eliminar 

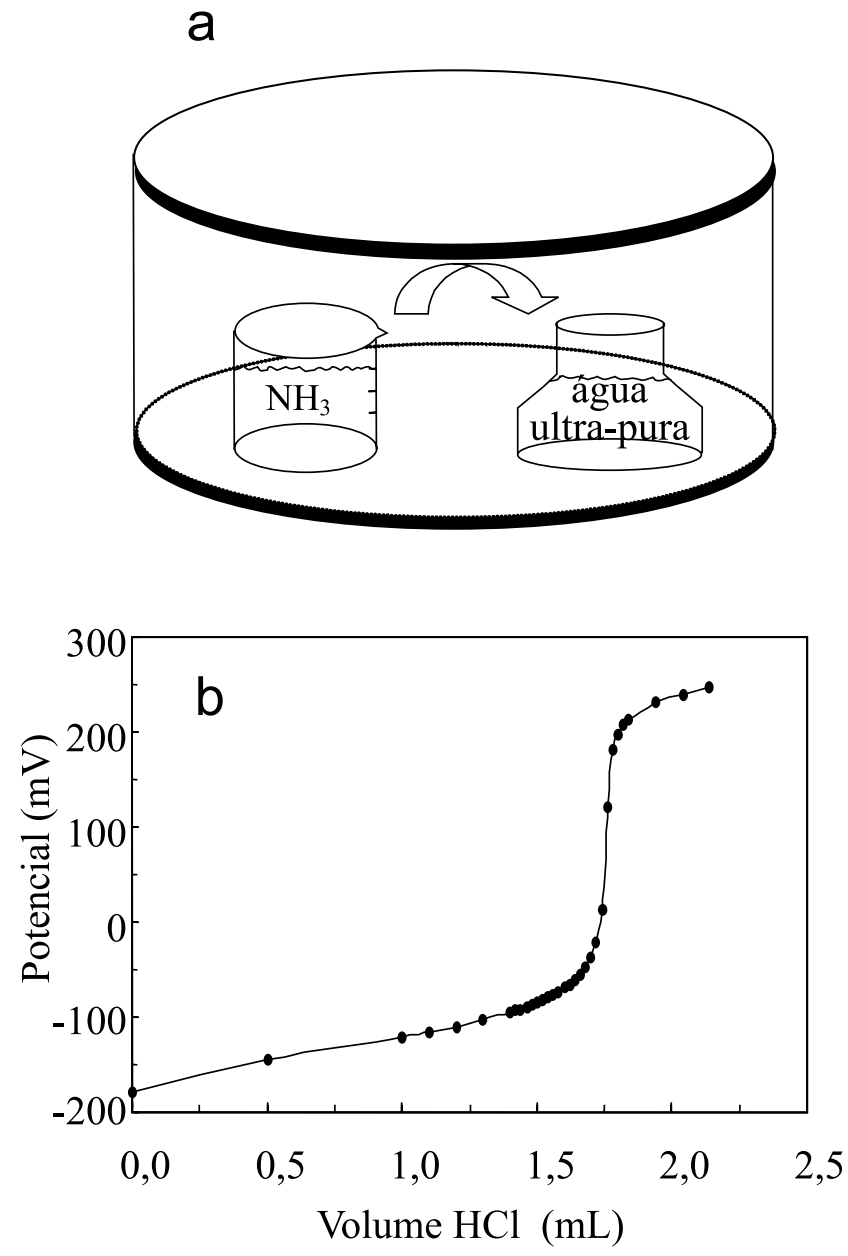

Figura 1. a) Desenho do sistema utilizado para destilação isotérmica da $\mathrm{NH}_{3}$ e b) titulação potenciométrica de uma alíquota $(20 \mu \mathrm{L})$ da solução destilada de amônia, com solução padronizada de $\mathrm{HCl}$ 9,99 $10^{-2} \mathrm{~mol} \mathrm{~L}^{-1}$

o excesso de acetato de amônio. A resina Chelex foi pipetada e transferida para a solução de tampão borato, que foi mantida sob agitação por $4 \mathrm{~h}$. A solução foi deixada em repouso por $1 \mathrm{~h}$ e o sobrenadante transferido para outro frasco. Ellis e Roberts ${ }^{20}$ relatam que há perda de eficiência da resina Chelex-100 na retenção de metais em $\mathrm{pH}$ acima de 7 , porém os autores se referem às soluções com elevadas concentrações de metais.

\section{Branco de reagentes e limite de detecção}

A avaliação sistemática do branco de reagentes durante todo o processo analítico é imprescindível para a aquisição de dados confiáveis. A Figura 2 ilustra a boa resolução dos voltamogramas para um branco de reagentes utilizando o ligante SA e 2 min de préconcentração, gerando um pico inicial de apenas 3,3 nA. Visto que o manuseio e a análise das amostras foram realizados em um laboratório comum, sem contar ao menos com uma bancada de fluxo laminar, os resíduos de cobre, chumbo e cádmio obtidos nos brancos listados na Tabela 2 podem ser considerados bastante satisfatórios.

Não houve diferença significativa entre as concentrações de cobre nos brancos usando o ligante $\mathrm{SA}$ ou oxina (teste-t, $\mathrm{P}=0,05$ ), indicando que é improvável que o cobre residual seja oriundo do ligante. As baixas concentrações de chumbo e cádmio no branco ilustram o eficiente controle na contaminação também para estes metais. A con-

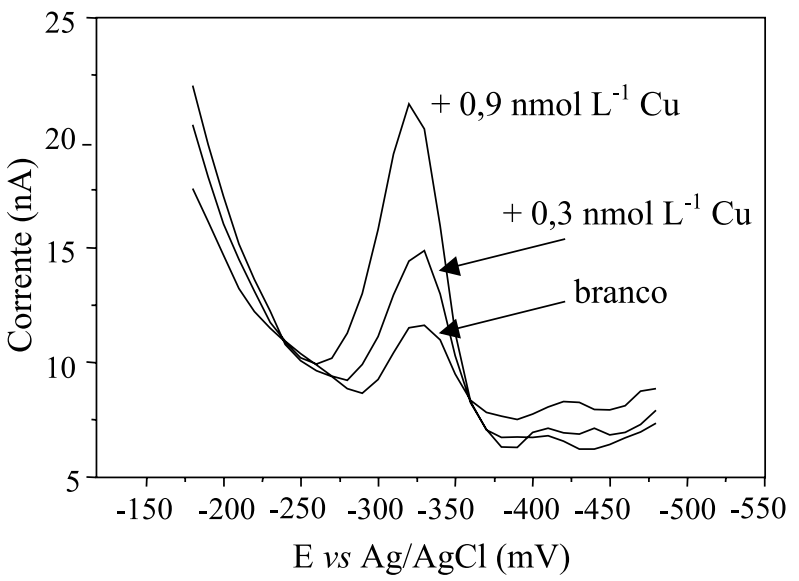

Figura 2. Voltamogramas obtidos na determinação de cobre em água ultrapura contendo 0,01 mol $L^{-1}$ tampão borato, $25 \mu \mathrm{mol} L^{-1} S A$ e usando $2 \mathrm{~min}$ de pré-concentração. As adições de padrão são indicadas, e a concentração de cobre encontrada foi de 0,35 $\mathrm{nmol} \mathrm{L}^{-1}$

centração de cobre obtida para um branco contendo 3 vezes mais tampão $\left(0,03 \mathrm{~mol} \mathrm{~L}^{-1}\right)$ ficou dentro do valor médio dos demais brancos, demonstrando a baixa contaminação da solução tampão. Apesar do mercúrio utilizado ter sido de alta pureza, é possível que este contribua para a concentração residual de cobre encontrada nos brancos de reagentes, assim como a água ultra-pura, instrumentação e manuseio. A eficiência na purificação tanto do $\mathrm{HCl}$ como da $\mathrm{NH}_{3}$ por destilação isotérmica foi testada em água ultra-pura adicionando as mesmas concentrações que aquelas utilizadas na acidificação e neutralização das amostras naturais. Antes da purificação dos reagentes, a concentração de cobre obtida foi de $30 \mathrm{nmol} \mathrm{L}^{-1}$, e depois da purificação $0,78 \pm 0,32(n=3)$, valor que não é significativamente diferente do branco na ausência de ácido e base (teste t, $\mathrm{P}=0,05$; Tabela 2). Portanto, a acidificação e posterior neutralização das amostras naturais puderam ser realizadas com segurança. Como as concentrações de cobre nos brancos de reagentes estiveram dentro do desvio padrão do método, não foi preciso corrigir os valores encontrados para as amostras.

O branco de campo tratou do manuseio de um frasco contendo água ultra-pura durante a amostragem de águas naturais como se fosse uma amostra (Tabela 2). Neste trabalho, dentro da embarcação, depois de realizadas algumas amostragens, a água ultra-pura de um frasco foi transferida para outro que foi esvaziado e manuseado

Tabela 2. Concentração de metais nos brancos de reagentes e brancos de campo em água ultra-pura

\begin{tabular}{ll}
\hline reagentes $\left(\mathrm{mol} \mathrm{L}^{-1}\right)$ & metal $\left(\mathrm{nmol} \mathrm{L}{ }^{-1}\right)$ \\
\hline 0,01 borato $+25 \times 10^{-6} \mathrm{SA}$ & {$[\mathrm{Cu}]=0,49 \pm 0,19(\mathrm{n}=7)$} \\
branco de campo - embarcação & {$[\mathrm{Cu}]=0,55$} \\
branco de campo - água de chuva & {$[\mathrm{Cu}]=0,28$ e 0,29} \\
0,01 borato $+20 \times 10^{-6}$ oxina & {$[\mathrm{Cu}]=0,54 \pm 0,23(\mathrm{n}=8)$} \\
& {$[\mathrm{Pb}]=0,40$} \\
& {$[\mathrm{Cd}]<0,41$} \\
0,03 borato $+20 \times 10^{-6}$ oxina & {$[\mathrm{Cu}]=0,42$} \\
0,01 borato $+20 \times 10^{-6}$ oxina & {$[\mathrm{Cu}]=0,78 \pm 0,32(\mathrm{n}=3)$} \\
$+0,02 \mathrm{HCl}+0,02 \mathrm{NH}_{3}$ & \\
\hline
\end{tabular}


como uma amostra. No caso do branco de campo para coleta de água de chuva, o funil acoplado ao frasco foi exposto no campo (campus da UFSC) por cerca de um minuto, visto que o tempo de exposição deve ser curto para evitar a deposição de material particulado da atmosfera e, em seguida, foi despejado sobre todo o diâmetro do funil $103 \mathrm{~mL}$ de água ultra-pura (volume médio de chuva coletado).

O limite de detecção obtido para o cobre foi de $0,18 \mathrm{nmol} \mathrm{L}^{-1}$ usando água ultra-pura, $0,01 \mathrm{~mol} \mathrm{~L}^{-1}$ tampão borato, $20 \mu \mathrm{mol} \mathrm{\textrm {L } ^ { - 1 }}$ oxina e 2 min de pré-concentração. Este foi calculado usando 3 vezes o desvio padrão ${ }^{8}$ de 10 varreduras $(5,91 \pm 0,42 \mathrm{nA})$, e dividindo este valor pela sensibilidade do método $\left(6,95 \mathrm{nA} \mathrm{nmol}^{-1} \mathrm{~L}^{-1}\right)$, determinada após a adição de padrões de cobre. Este limite de detecção é um pouco maior que aquele obtido por Campos e van den Berg ${ }^{12}$ que usaram 1 min de pré-concentração e água do mar purificada $\left(0,2 \mathrm{nmol} \mathrm{L}^{-1}\right)$, porém, pode ser abaixado utilizando um maior tempo de pré-concentração. Entretanto, este procedimento não foi necessário, visto que o limite de detecção obtido neste trabalho supera as necessidades para os fins aqui propostos.

\section{Filtração das amostras}

A filtração das amostras é indispensável quando se pretende avaliar apenas a fração solúvel do metal, sendo este procedimento mais uma possível fonte de contaminação. Neste trabalho foi utilizado um sistema de filtração fechado de polissulfona (Sartorius, capacidade de $250 \mathrm{~mL}$ ) e membranas de acetato de celulose de poro 0,45 $\mu \mathrm{m}$ (Millipore). A filtração foi realizada sob pressão de nitrogênio, pois este procedimento é muito mais limpo que o uso de vácuo.

O sistema de filtração foi inicialmente lavado com detergente diluído, enxaguado com água desionizada, $\mathrm{HCl} 1 \mathrm{~mol} \mathrm{~L}^{-1}$ e novamente enxaguado com grandes quantidades de água desionizada. $\mathrm{O}$ uso de ácidos mais concentrados não é aconselhável pois estes podem danificar os anéis de borracha do sistema de filtração. Entre a filtração de uma amostra e outra, a limpeza do sistema foi feita utilizando a solução ácida e água desionizada. Uma primeira alíquota de cerca de $30 \mathrm{~mL}$ de cada amostra filtrada foi utilizada para enxaguar o sistema, sendo em seguida desprezada.

A possível contaminação pela membrana de filtro sem tratamento prévio foi avaliada filtrando sucessivamente duas alíquotas de 50 $\mathrm{mL}$ de água ultra-pura, e avaliando a concentração de cobre em cada uma delas. Na primeira alíquota filtrada a concentração de cobre foi de $1,1 \mathrm{nmol} \mathrm{L}^{-1}$, aumentando para $1,9 \mathrm{nmol} \mathrm{L}^{-1}$ na segunda fração; o que sugere uma elevada contaminação pela membrana de filtro, visto que a concentração inicial de cobre na água ultra-pura era de apenas $0,52 \pm 0,21 \mathrm{nmol} \mathrm{L}^{-1}(\mathrm{n}=15)$. Um novo filtro foi introduzido no sistema de filtração, sendo filtrados $50 \mathrm{~mL}$ de $\mathrm{HCl} 1 \mathrm{~mol} \mathrm{~L}^{-1}$ e 100 $\mathrm{mL}$ de água ultra-pura, que foram desprezados. Posteriormente, duas alíquotas de $50 \mathrm{~mL}$ de água ultra-pura foram filtradas, reservando cada uma delas. A concentração de cobre em cada uma das frações foi de 0,42 e $0,44 \mathrm{nmol} \mathrm{L}^{-1}$ respectivamente, valores que não diferem da concentração inicial de cobre na água ultra-pura, evidenciando a eficiência na pré-lavagem dos filtros. Pequenos volumes foram filtrados usando um sistema compacto de filtração de polipropileno de $25 \mathrm{~mm}$ de diâmetro, utilizando seringas plásticas descartáveis para introdução das amostras. O procedimento de limpeza do filtro consistiu em filtrar $20 \mathrm{~mL}$ de $\mathrm{HCl} 1 \mathrm{~mol} \mathrm{~L}^{-1}, 30 \mathrm{~mL}$ de água desionizada e $10 \mathrm{~mL}$ da própria amostra, que foram desprezados.

\section{Água do mar certificada e amostras naturais}

Somente após a obtenção de brancos satisfatórios comprovando o controle da contaminação e pureza dos reagentes é que foi realizada a determinação de cobre dissolvido total (após irradiação com
UV) na amostra de água do mar certificada CRM-403. A Figura 3 mostra os voltamogramas obtidos para a análise de cobre em uma alíquota do material certificado e a determinação da concentração do metal na amostra. A concentração média e desvio padrão de cobre em duas ocasiões distintas de análise, com cerca de 6 meses de intervalo foram $4,17 \pm 0,85(\mathrm{n}=3)$ e $3,70 \pm 0,86 \mathrm{nmol} \mathrm{L}^{-1}(\mathrm{n}=6)$. Os valores médios obtidos estão dentro do valor certificado de 4,00 $\pm 0,38$ nmol L ${ }^{-1}$, o que mostra a boa exatidão do método, e o bom controle da contaminação, mesmo em um laboratório comum. O desvio padrão obtido de cerca de $20 \%$ pode ser considerado bastante satisfatório e indica que este deve ser o desvio padrão esperado para amostras naturais contendo cerca de $4,0 \mathrm{nmol} \mathrm{L^{-1 }} \mathrm{Cu}$.

A análise de materiais certificados é, sem dúvida, o meio mais simples e rápido de testar a exatidão e precisão do método de análise, porém, o elevado custo destes materiais pode dificultar que o analista se assegure regularmente sobre a qualidade de seus resultados. Neste trabalho, logo após as análises satisfatórias de brancos e da água do mar certificada, foi realizada a coleta de 1 litro de água do mar na costa de Florianópolis (Praia de Santo Antônio de Lisboa). A amostra foi imediatamente levada ao laboratório, filtrada, homogeneizada em frasco de 1 litro, acidificada, e submetida à aná-
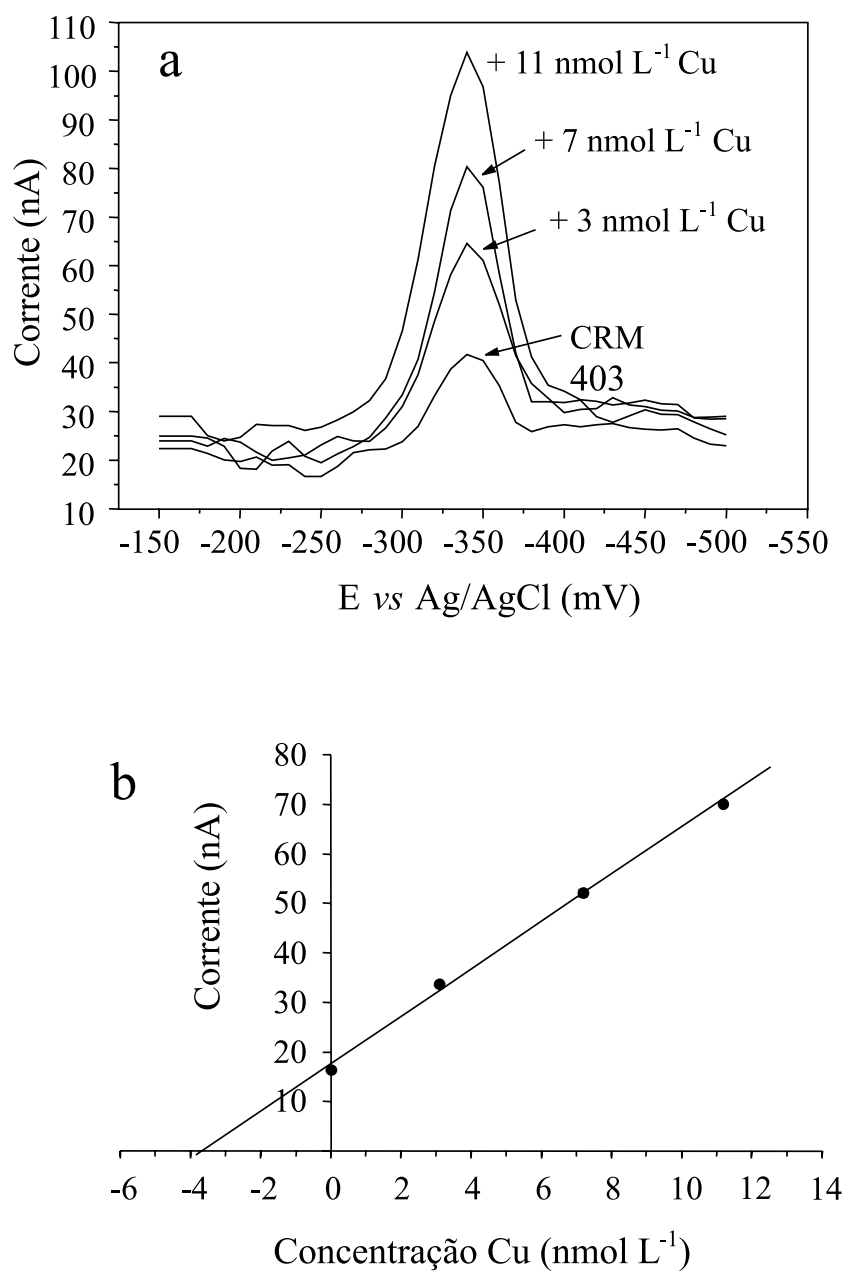

Figura 3. a) Voltamogramas obtidos para a determinação da concentração de cobre na água do mar certificada CRM-403. b) Corrente de pico de acordo com a concentração de cobre adicionado na amostra, mostrando a reta gerada através da regressão linear dos pontos para determinação da concentração de cobre 
lise de cobre após $4 \mathrm{~h}$ de irradiação ultravioleta, sempre seguindo todas as recomendações já descritas. A concentração média e desvio padrão de cobre dissolvido total encontrados para 5 determinações foram de 4,38 $\pm 0,75 \mathrm{nmol} \mathrm{L}^{-1}$. O restante da amostra foi preservado em geladeira, sendo esta chamada de "amostra certificada secundária”. Sua concentração de cobre foi reavaliada periodicamente para uma simples auto-avaliação do analista e após a preparação de novos padrões. No caso de ser necessário avaliar a concentração de uma gama de metais, pode-se coletar um volume maior de amostra. O uso desse tipo de amostra não substitui a amostra certificada, mas pode ser muito útil para a realização de pré-testes.

A Tabela 3 traz alguns exemplos da especiação de cobre em amostras naturais, com o objetivo de ilustrar que só foi possível obter estes resultados devido ao rígido procedimento analítico aqui descrito, desde a amostragem, o manuseio, até a análise do metal. Estes exemplos também ilustram a importância do controle da contaminação para uma diversidade de ambientes, incluindo água de chuva, onde a concentração de cobre dissolvido total atingiu valores tão baixos quanto 1,5 e $2,0 \mathrm{nmol} \mathrm{L}^{-1}$, mas ainda assim foi possível determinar a fração lábil do metal.

Tabela 3. Especiação de cobre (concentração em nmol L $\mathrm{L}^{-1}$ ) para amostras de águas naturais de Florianópolis, SC, incluindo uma amostra de Urussanga, SC. Cobre dissolvido total refere-se à amostra acidificada e irradiada para destruição da matéria orgânica, enquanto cobre lábil é aquele avaliado na amostra apenas filtrada, e sem qualquer outro tratamento. A concentração do cobre complexado pela matéria orgânica foi calculada pela diferença entre as outras duas espécies

\begin{tabular}{lccc}
\hline amostra & $\begin{array}{c}\text { Cu dissolvido } \\
\text { total }\end{array}$ & $\begin{array}{c}\mathrm{Cu} \\
\text { lábil }\end{array}$ & $\begin{array}{c}\mathrm{Cu} \\
\text { orgânico }\end{array}$ \\
\hline água de chuva & 1,5 & 0,2 & 1,3 \\
$01 / 02 / 00$ & 2,0 & 1,4 & 0,6 \\
$16 / 03 / 00$ & 6,9 & 2,5 & 4,4 \\
$17 / 07 / 00$ (Urussanga) & 4,4 & 2,4 & 2,0 \\
\hline Praia Sto Antonio de Lisboa & 3,0 & 1,3 & 1,7 \\
\hline Rio João Gualberto & & &
\end{tabular}

\section{CONCLUSÕES}

Visto que a alta sensibilidade das técnicas analíticas já é um requisito disponível para determinação de metais traços em águas naturais, provavelmente um dos maiores desafios hoje é evitar a contaminação das amostras, desde a coleta até a análise. Portanto, a purificação de reagentes, análise freqüente de brancos e de amostras certificadas, cuidados especiais na amostragem e manuseio estão entre os requisitos indispensáveis para a obtenção de dados consistentes sobre a biogeoquímica de metais no meio ambiente. A observação desses requisitos, descritos em detalhe neste trabalho, assegurou o baixo nível de cobre residual nos reagentes e em todo o processo de manuseio e análise das amostras. Os baixos valores de chumbo e cádmio nos brancos de reagentes demonstraram o bom controle da contaminação também para esses metais.
Os métodos alternativos aqui discutidos de purificação de água contaminada com cobre usando uma suspensão de $\mathrm{MnO}_{2}$, e a destilação do $\mathrm{HCl}$ e $\mathrm{NH}_{3}$ em um recipiente plástico, ao invés do tradicional destilador de quartzo, demonstraram ter grande eficiência na remoção de metais, além de serem métodos muito simples e baratos.

Algumas publicações a respeito da certificação de amostras de água do mar e de exercícios de calibração, ${ }^{9,11}$ ilustram a sofisticação tecnológica dos laboratórios envolvidos. Porém, este trabalho vem demonstrar que é possível realizar análises de metais no nível de traço e ainda avaliar sua especiação química em amostras de ambientes costeiros e de águas de chuva, em um laboratório comum, sem mesmo uma bancada de fluxo laminar e com o mínimo de investimento financeiro.

\section{AGRADECIMENTOS}

Os autores são gratos ao CNPq pela concessão da bolsa de Pesquisadora Visitante para M. L. A. M. Campos, à CAPES por conceder as bolsas de mestrado aos alunos A. Bendo e F. C. Viel, aos professores A. Spinelli e D. R. Zanette por ceder os equipamentos indispensáveis para realização do trabalho, à Pós graduação em Química da UFSC pelo apoio financeiro, e à EPAGRI de Urussanga pela coleta das amostras de chuva naquela cidade.

\section{REFERÊNCIAS}

1. Widdows, J.; Donkin, P. Em The Mussel Mytilus: Ecology, Physiology, Genetics and Culture; Gosling E., ed.; Elsevier: Amsterdam, 1992, cap. 8.

2. Kennish, M. J.; Practical Handbook of Estuarine and Marine Pollution, CRC Press: Marine Science Series, 1996.

3. Libes, S. M.; An Introduction to Marine Biogeochemistry, Wiley: New York, 1992.

4. Boyle, E. A.; Sclater, F. R.; Edmond, J. M.; Earth Planet. Sci. Lett. 1977, 37,38 .

5. Schaule, B. K.; Patterson, C. C.; Earth Planet. Sci. Lett. 1981, 54, 97.

6. Florence, T. M.; Batley, G. E.; Crit. Rev. Anal. Chem. 1980, 9, 219.

7. Benoit. G.; Hunter, K. S.; Rozan, T. F.; Anal. Chem. 1997, 69, 1006.

8. Batley, G.E.; Mar. Pollut. Bull. 1999, 39, 23.

9. Landing, W. M.; Cutter, G. A.; Dalziel, J. A.; Flegal, A. R.; Powell, R. T.; Schmidt, D.; Shiller, A.; Statham, P.; Westerlund, S.; Resing, J.; Mar. Chem. 1995, 49, 253.

10. Quevauviller, Ph.; Kramer, K. J. M.; van der Vlies, E. M.; Vercoutere, K.; Griepink, B.; Mar. Pollut. Bull. 1992, 24, 33.

11. van den Berg, C. M. G. Em Chemical Oceanography; Riley, J. P., ed; Academic Press: London, 1988, cap. 51.

12. Campos, M. L. A. M.; van den Berg, C. M. G.; Anal. Chim. Acta 1994, 284,481 .

13. van den Berg, C. M. G.; J. Electroanal. Chem. 1986, 215, 111.

14. Campos, M. L. A. M.; Mello, L. C.; Zanette, D. R.; Sierra, M. M. de S.; Bendo, A.; Quim. Nova 2001, 24, 257.

15. Statham, P. J.; Howard, A. G.; Inorganic Trace Analysis Philosophy and Practice, Wiley: New York, 1993.

16. Campos, M. L. A. M.; Tese de Doutorado, Universidade de Liverpool, Reino Unido, 1992.

17. Achterberg. E. P.; Tese de Doutorado, Universidade de Liverpool, Reino Unido, 1993.

18. Chester, R.; Marine Geochemistry, Unwin Hyman: London, 1990.

19. Achterberg, E. P.; van den Berg, C. M. G.; Boussemart, M.; Davison, W.; Geochim. Cosmochim. Acta 1997, 61, 5233.

20. Ellis, L. A.; Roberts, D. J.; J. Anal. At. Spectrom. 1998, 13, 631.

21. Cornelis, R.; Dyg., S.; Griepink, B.; Dams. R.; Fresenius'J. Anal. Chem. 1990, 338, 414 . 